\title{
Stage 1 Registered Report: Anomalous perception in a Ganzfeld condition - A meta-analysis of more than $\mathbf{4 0}$ years investigation [version 1; peer review: awaiting peer review]
}

\author{
Patrizio E. Tressoldi (D) 1, Lance Storm² \\ ${ }^{1}$ Science of Consciousness Research Group, Università degli studi di Padova, Padova, ITALY, 35131, Italy \\ ${ }^{2}$ School of Psychology, University of Adelaide, Adelaide, Australia, 5005, Australia
}

V1 First published: $30 \mathrm{Jul}$ 2020, 9:826

https://doi.org/10.12688/f1000research.24868.1

Latest published: $30 \mathrm{Jul}$ 2020, 9:826

https://doi.org/10.12688/f1000research.24868.1

\section{Open Peer Review \\ Reviewer Status AWAITING PEER REVIEW}

Any reports and responses or comments on the article can be found at the end of the article.

\section{Abstract}

This meta-analysis is an investigation into anomalous perception (i.e., conscious identification of information without any conventional sensorial means). The technique used for eliciting an effect is the ganzfeld condition (a form of sensory homogenization that eliminates distracting peripheral noise). The database consists of peer-reviewed studies published between January 1974 and June 2020 inclusive. The overall effect size will be estimated using a frequentist model and a Bayesian random model. Moderator analysis will be used to examine the influence of level of experience of participants and the type of task. Publication bias will be estimated by using three different tests. Trend analysis will be conducted on the cumulative database.

Keywords

meta-analysis, ganzfeld; anomalous cognition, publication bias;

consciousness

Corresponding author: Patrizio E. Tressoldi (patrizio.tressoldi@unipd.it)

Author roles: Tressoldi PE: Conceptualization, Data Curation, Formal Analysis, Investigation, Methodology, Software, Writing - Original Draft Preparation; Storm L: Conceptualization, Data Curation, Methodology, Supervision, Writing - Original Draft Preparation, Writing - Review \& Editing

Competing interests: No competing interests were disclosed.

Grant information: The author(s) declared that no grants were involved in supporting this work.

Copyright: ( 2020 Tressoldi PE and Storm L. This is an open access article distributed under the terms of the Creative Commons Attribution License, which permits unrestricted use, distribution, and reproduction in any medium, provided the original work is properly cited.

How to cite this article: Tressoldi PE and Storm L. Stage 1 Registered Report: Anomalous perception in a Ganzfeld condition - A meta-analysis of more than $\mathbf{4 0}$ years investigation [version 1; peer review: awaiting peer review] F1000Research 2020, 9:826 https://doi.org/10.12688/f1000research.24868.1

First published: $30 \mathrm{Jul}$ 2020, 9:826 https://doi.org/10.12688/f1000research.24868.1 


\section{Introduction}

The possibility of identifying pictures or video clips without conventional (sensorial) means, in a ganzfeld environment, is a decades old controversy, dating back to the pioneering investigation of Charles Honorton, William Braud and Adrian Parker between 1974 and 1975 (Parker, 2017).

In the ganzfeld, a German term meaning 'whole field', participants are immersed in an homogeneous sensorial field were peripheral visual information is masked out by red light diffused by translucent hemispheres (often split halves of ping-pong balls or special glasses) placed over the eyes, while a relaxing rhythmic sound, or white or pink noise, is fed through headphones to shield out peripheral auditory information. Once participants are sensorially isolated from external visual and auditory stimulation, they are in a favourable condition for producing inner mental contents about a randomly-selected target hidden amongst decoys. The mentation they produce can either be used by the participant to guide his/her target selection, or it can be used to assist in an independent judging process.

In the prototypical procedure, participants are tested in a room isolated from external sounds and visual information. After they made themselves comfortable in a reclining armchair, they receive the instructions related their task during the ganzfeld condition. Even if there are different verbatim versions, the instructions describe what they should do mentally in order to detect the information related to the target and how to filter out the mental contents not related to it. This information will be described aloud and recorded for playback before or during the target identification phase. After the relaxation phase, they are exposed to the ganzfeld condition for a period ranging from 15 to 30 minutes. During this phase, participants describe verbally all images, feelings, emotions, they deem related to the target usually a picture or a short videoclip of real objects or events.

Once the ganzfeld phase is completed, participants are presented with our choices (the target plus three decoys) of the same format, e.g. picture or videoclip, and they must choose which one is the target (binary decision). Alternatively, they may be asked to rate all four (e.g., from 0 to 100), to indicate the strength of relationship between the information detected during the ganzfeld phase and the images or video clips contents.

A variant of the judgment phase is to send the recording of the information retrieved during the ganzfeld phase to an external judge for independent ratings of the target. In order to prevent voluntary or involuntary leakage of information about the target by the experimenters, the research assistant who interact with the participants must be blind to the target identity until the participants' rating task is over. The choice of the target and the decoys is usually made using automatic random procedures, and scores are automatically fed onto a scoring sheet.
There are three different ganzfeld conditions:

- Type 1: the target is chosen after the judgment phase;

- Type 2: the target is chosen before the ganzfeld phase;

- Type 3: the target is chosen before the ganzfeld phase and presented to a partner of the participant isolated in a separate and distant room.

These differences are related to some theoretical and perceptual concepts we will discuss later. It is important to note that type of task makes no difference to the participant who only engages in target identification after the ganzfeld phase.

\section{Review of the Ganzfeld Meta-Analyses}

It is interesting to note that most of the cumulative findings (meta-analyses) of this line of investigation were periodically published in the mainstream journal Psychological Bulletin.

Honorton (1985) undertook one of the first meta-analyses of the many ganzfeld studies completed by the mid-1980s. In total, 28 studies yielded a collective hit rate of 38\%, where mean chance expectation (MCE) was $25 \%$. Various flaws in his approach were pointed out by Hyman (1985), but in their joint-communiqué they agree that "there is an overall significant effect in this database that cannot reasonably be explained by selective reporting or multiple analysis" (Hyman \& Honorton, 1986, p. 351).

A second major meta-analysis on a set of 'autoganzfeld' studies was performed by Bem \& Honorton (1994). These studies followed the guidelines laid down by Hyman \& Honorton (1986). Moreover the autoganzfeld procedure avoids methodological flaws by using a computer-controlled target randomization, selection, and judging technique. They overall reported hit rate of $32.2 \%$ exceeded again the mean chance expectation.

Milton \& Wiseman (1999) meta-analysed further 30 studies collected for the period 1987 to 1997; reporting an overall nonsignificant standardized effect size of 0.013. However, Jessica Utts (personal communication, December 11, 2009) using the exact binomial test on trial counts only $(N=1198$; Hits $=327)$, found a significant hit rate of $27 \%(p=0.036)$.

Storm \& Ertel (2001) comparing Milton \& Wiseman's (1999) database with Bem \& Honorton's (1994) one, found the two did not differ significantly. Furthermore Storm and Ertel went on to compile a 79-study database, which had a statistically significant mean standardized effect size of 0.138 .

Storm et al. (2010), meta-analysed a database of 29 ganzfeld studies published during the period 1997 to 2008, yielding a standardized effect size of 0.14. Rouder et al. (2013) reassessing Storm et al.'s (2010) meta-analysis, with a Bayesian meta-analysis found evidence for the existence of an anomalous perception in the original dataset observing a 
Bayes Factor of 330 in support to the alternative hypothesis (p. 241). However they contended the effect could be due to "difficulties in randomization" (p. 241), arguing that ganzfeld studies with computerized randomization had smaller effects than those with manual randomization. The reanalysis by Storm et al.'s (2013) showed that this conclusion was unconvincing as it was based on Rouder et al.'s faulty inclusion of different categories of study.

In the last meta-analysis by Storm \& Tressoldi (2020), related to the studies published from 2008 to 2018, the overall standardized effect size was $0.133 ; 95 \%$ CI: $0.06-0.18$.

\section{This study}

The main aim of this study is to meta-analyse all available ganzfeld studies dating from 1974 up to June 2020 in order to assess the overall effect size of the database and determine whether there are moderator variables that affect task performance; in particular, we hypothesize that participant type and type of task are two major moderators of effect size (see Methods section).

\section{Methods}

Reporting guidelines

This study will follow the guidelines of the APA Meta-Analysis Reporting Standard (Appelbaum et al., 2018) and the Preferred Reporting Items for Systematic Review and Meta-Analysis Protocols (PRISMA-P, Moher et al., 2015).

\section{Studies retrieval}

Retrieval of studies related to anomalous perception in a Ganzfeld environment is simplified, firstly by the fact that most of these studies have already been retrieved for previous meta-analyses, as cited in the introduction. Secondly, this line of investigation is carried out by a small community of researchers. Thirdly, most of the studies of interest to us are published in specialized journals that adopted the editorial policy of accepting paper with results that are statistically non-significant (according to the frequentist approach). This last condition is particularly relevant because it reduces the publication bias due to the non-rejection of the statistical null hypothesis often consequent to reduced statistical power. However, in order to integrate the previous retrieval method, we will carry-out an online search with Google Scholar, PubMed and Scopus databases of all papers from 1974 to 2020 including in the title and/or the abstract the word "ganzfeld" (e.g. for PubMed: Search: ganzfeld[Title/Abstract] Filters: from 1974 - 2020).

\section{Studies inclusion criteria}

The following inclusion criteria will be adopted:

- Studies related to anomalous perception in a ganzfeld environment;

- Studies must use human participants only (not animals);

- Number of participants must be in excess of two to avoid the inherent problems that are typical in case studies;
- Target selection must be randomized by using a Random Number Generator (RNG) in a computer or similar electronic device, or a table of random numbers. Randomization procedures must not be manipulated by the experimenter or participant;

- Studies must provide sufficient information (e.g., number of trials and outcomes) for the authors to calculate the direct hit-rates and effect size values, so that appropriate statistical tests can be conducted.

- Peer reviewed studies even if published in proceedings;

\section{Variables coding}

For each included study, one of the authors, expert in meta-analyses, will code the following variables:

- Authors;

- Year of publication;

- Number of trials;

- Number of hits;

- Number of choices of each trial;

- Task type (Type 1,2 or 3);

- Participants type (selected vs. unselected). The authors of the study will score as selected all participants that were screened for one or more particular characteristic deemed favourable for the performance in this type of task.

The second author will randomly check $10 \%$ of all studies, and the data will be compared with those extracted by the other author. Discrepancies will be corrected by inspecting the original papers.

The complete database will be made available through open access posting within the dedicated project in the Open Science Framework (https://osf.io/t7sya/) platform.

\section{Effect size measures}

As standardized measure of effect size, we will use the following formula: Binomial $\mathrm{Z}$ score $/ \sqrt{ }$ number of trials. The Binomial $\mathrm{Z}$ score will be obtained applying the formula implemented online at http://vassarstats.net/binomialX.html

In order to take in account the effect size overestimation bias in small samples, the effect size will be transformed in the Hedge's $g$ effect size, obtaining the corresponding variance by applying the formula presented in Borenstein et al. (2009, pp. 27-28).

\section{Overall effect size estimation}

The overall effect size estimation of the whole database will be calculated by applying both a frequentist and a Bayesian random model for testing its robustness. 


\section{Frequentist random model}

Following the recommendations of Langan et al. (2019), we will use the restricted maximum likelihood (REML) approach to estimate the heterogeneity variance with the Knapp and Hartung method for adjustment to the standard errors of the estimated coefficients (Rubio-Aparicio et al., 2018).

Furthermore, in order to control for possible influence of outliers, we will calculate the median and mode of the overall effect size applying the method suggested by Hartwig et al. (2020).

These calculations will be implemented in the $\mathrm{R}$ statistical environment with the metafor package v. 2.4 (Viechtbauer, 2017). See syntax provided as extended data (Tressoldi \& Storm, 2020).

\section{Bayesian random model}

As priors for the overall effect size we will use a normal distribution with Mean $=0.01 ; S D=0.03$, constrained positive, lower bound $=0$ (Rouder et al., 2019), given our expectation of a positive value. This Bayesian meta-analysis will be implemented with the MetaBMA package v. 0.6.3 (Heck et al., 2017). See Syntax in the Supporting Information.

\section{Publication bias tests}

Following the suggestions of Carter et al. (2019), we will apply three tests to assess publication bias:

- the 3-parameter selection model (3PSM), as implemented by Coburn \& Vevea (2019) with the package 'weightr' v.2.0.2;

- the p-uniform* (star) v. 0.2.2 test as described by van Aert \& van Assen (2019), and

- the sensitivity analysis using the Mathur \& VanderWeele (2020) package PublicationBias v.2.2.0.

The three parameters model represent the average true underlying effect, $\delta$; the heterogeneity of the random effect sizes, $\tau^{2}$; and the probability that there is a nonsignificant effect in the pool of effect sizes. The probability parameter is modeled by a step function with a single cut point at $p=0.025$ (one-tailed), which corresponds to a two-tailed $p$ value of 0.05 . This cut point divides the range of possible $p$ values into two bins: significant and nonsignificant. The three parameters are estimated using maximum likelihood (Carter et al., 2019, p. 124).

The $p$-uniform* test, is an extension and improvement of the $p$-uniform method. P-uniform* improves upon $p$-uniform giving a more efficient estimator avoiding the overestimation of effect size in case of between-study variance in true effect sizes, thus enabling estimation and testing for the presence of between-study variance in true effect sizes.

Sensitivity analysis as implemented by Mathur \& Vander-Weele (2020), assumes a publication process such that "statistically significant" results are more likely to be published than negative or "nonsignificant" results by an unknown ratio, $\eta$ (eta). Using inverse-probability weighting and robust estimation that accommodates non-normal true effects, small meta-analyses, and clustering, it enables statements such as: "For publication bias to shift the observed point estimate to the null, 'significant' results would need to be at least 30-fold more likely to be published than negative or 'nonsignificant' results" (p. 1). Comparable statements can be made regarding shifting to a chosen non-null value or shifting the confidence interval. See Syntax in the Supporting Information

\section{Cumulative meta-analysis}

In order to study the overall trend of the cumulative evidence and in particular for testing the presence of a decline or incline effect, we will perform a cumulative effect size estimation (see Syntax in the Supporting Information)

\section{Moderators effects}

We will compare the influence of the following two moderators: (i) Type of participant, and (ii) Type of task.

As described in the Variable Coding paragraph, the variable Type of participant, will be coded in a binary way: selected vs unselected. Type of task will be coded as Type 1, Type 2, and Type 3, as described in the Introduction. See Syntax in the Supporting Information.

\section{Statistical power}

Once the overall effect size and its precision are estimated, we will calculate the number of trials necessary to achieve a statistical power of at least .80 with an $\alpha=.05$. With this estimation we can examine how many studies in the database reached this threshold.

\section{Reporting}

The search and selection of the studies will be presented by using a PRISMA flowchart.

\section{Descriptive statistics}

Descriptive statistics will be produced related to the variables, trials, hits, participant type, and task types.

\section{Overall effect size}

We will present the estimated average effect size along with the corresponding 95\% Confidence Intervals or Credible Intervals of both the Frequentist and Random Models as described in the Methods section. We will calculate the values of $\tau^{2}$ and $\mathrm{I}^{2}$ (Higgins \& Thompson, 2002), and their confidence intervals, as measures of between-study variance.

\section{Publication bias tests}

We will present the results of the three publication bias tests described in the Methods section.

\section{Cumulative effect size}

The results of the cumulative meta-analysis will be represented with a cumulative forest plot. 


\section{Moderator effects}

We will present and compare the average effect size along with the corresponding 95\% Confidence Intervals of the two types the participant, and of the three task types.

Dissemination of information

Apart the Registered Report, all information related to this study will be made available open access at Open Science Framework.

Study status

The study has not started yet.

\section{Discussion}

We will discuss the robustness of the overall results in order to determine a degree of confidence in the evidence for anomalous perception. In case of an insufficient degree of confidence in the evidence, we will consider whether it is worthwhile pursuing such a line of investigation and offer solutions to improve the evidence.

However, even if the overall results show a sufficient degree of evidence, we will discuss how this line of investigation can instil greater confidence by using a preregistration registry as proposed by Watt \& Kennedy (2016) in order to reduce so-called questionable research practices (John et al., 2012), and provide more transparent procedures during data collection and analysis (see for example, the Transparent Psi Project; Kekecs et al., 2019).

\section{Data availability}

Underlying data

No data are associated with this article

\section{Extended data}

Figshare: Anomalous perception in a gazfeld condition: a meta-analysis of more than 40 years of investigation. https://doi.org/ 10.6084/m9.figshare.12674618.v1 (Tressoldi \& Storm, 2020)

- Syntax Details.docx (Syntax related to all statistical analyses)

\section{Reporting guidelines}

Figshare: PRISMA-P checklist for 'Stage 1 Registered Report: Anomalous perception in a Ganzfeld condition - A meta-analysis of more than 40 years investigation' https://doi. org/10.6084/m9.figshare.12674618.v1 (Tressoldi \& Storm, 2020)

Data are available under the terms of the Creative Commons Attribution 4.0 International license (CC-BY 4.0).
Appelbaum M, Cooper H, Kline RB, et al.: Journal article reporting standards for quantitative research in psychology: The APA Publications and Communications Board task force report. Am Psychol. 2018; 73(1): 3-25. PubMed Abstract | Publisher Full Text

Bem DJ, Honorton C: Does psi exist? Replicable evidence for an anomalous process of information transfer. Psychol Bull. 1994; 115(1): 4-18. Publisher Full Text

Borenstein M, Hedges LV, Higgins JPT, et al:: Introduction to Meta-Analysis. Chichester, UK: John Wiley \& Sons, Ltd. 2009.

Publisher Full Text

Carter E, Schönbrodt F, Gervais W, et al:: Correcting-bias-in-psychology. Adv Methods Pract Psychol Sci. 2019; 2(2): 115-144.

Publisher Full Text

Coburn KM, Vevea JL: Package 'weightr'. Estimating Weight-Function Models for Publication Bias. 2019

Reference Source

Hartwig FP, Smith GD, Schmidt AF, et al:: The median and the mode as robust meta-analysis estimators in the presence of small-study effects and outliers. Res Synth Methods. 2020; 11(3): 397-412.

PubMed Abstract | Publisher Full Text | Free Full Text

Heck DW, Gronau QF, Wagenmakers E: metaBMA: Bayesian model averaging for random and fixed effects meta-analysis. 2017.

Publisher Full Text

Higgins JPT, Thompson SG: Quantifying heterogeneity in a meta-analysis. Stat Med. 2002; 21(11): 1539-1558.

PubMed Abstract | Publisher Full Text

Hyman R: The ganzfeld psi experiment: A critical appraisal. $J$ Parapsychol. 1985; 49(1): 3-49.

Reference Source

Hyman R, Honorton C: Joint communiqué: The psi ganzfeld controversy.

J Parapsychol. 1986; 50(4): 351-364.

Reference Source

Honorton C: Meta-analysis of psi ganzfeld research: A response to Hyman. $J$ Parapsychol. 1985; 49(1): 51-91.

Reference Source

John LK, Loewenstein G, Prelec D: Measuring the Prevalence of Questionable
Research Practices With Incentives for Truth Telling. Psychol Sci. 2012; 23(5): 524-532.

PubMed Abstract | Publisher Full Text

Kekecs Z, Aczel B, Palfi B, et al.: Raising the value of research studies in psychological science by increasing the credibility of research reports: The

Transparent Psi Project - Preprint. 2019.

Publisher Full Text

Langan D, Higgins JPT, Jackson D, et al.: A comparison of heterogeneity variance estimators in simulated random-effects meta-analyses. Res Synth Methods. 2019; 10(1): 83-98.

PubMed Abstract | Publisher Full Text

Mathur MB, VanderWeele TJ: Sensitivity analysis for publication bias in metaanalyses [preprint]. 2020.

Reference Source

Milton J, Wiseman R: Does psi exist? Lack of replication of an anomalous process of information transfer. Psychol Bull. 1999; 125(4): 387-391. PubMed Abstract | Publisher Full Text

Moher D, Stewart L, Shekelle $P$, et al:: Preferred reporting items for systematic review and meta-analysis protocols (PRISMA-P) 2015 statement. Syst Rev. 2015; 4(1): 1

PubMed Abstract | Publisher Full Text | Free Full Text

Parker A: 'Ganzfeld'. Psi Encyclopedia. London: The Society for Psychical Research. 2017.

Reference Source

Rouder JN, Morey RD, Province JM: A Bayes factor meta-analysis of recent extrasensory perception experiments: Comment on Storm, Tressoldi, and Di Risio (2010). Psychol Bull. 2013; 139(1): 241-247.

PubMed Abstract | Publisher Full Text

Rouder JN, Haal JM, Davis-Stober CP, et al.: Beyond overall effects: A Bayesian approach to finding constraints in meta-analysis. Psychol Methods. 2019; 24(5): 606-621.

PubMed Abstract | Publisher Full Text

Rubio-Aparicio M, López-López JA, Sánchez-Meca J, et al.: Estimation of an overall standardized mean difference in random-effects meta-analysis if the distribution of random effects departs from normal. Res Synth Methods. 2018; 9(3): 489-503.

PubMed Abstract | Publisher Full Text 
Storm L, Ertel S: Does psi exist? Comments on Milton and Wiseman's (1999) meta-analysis of ganzfeld research. Psychol Bull. 2001; 127(3): 424-433, discussion 434-8.

PubMed Abstract | Publisher Full Text

Storm L, Tressoldi PE, Di Risio L: Meta-analyses of free-response studies, 1992-2008: Assessing the noise reduction model in parapsychology. Psychol Bull. 2010; 136(4): 471-485.

PubMed Abstract | Publisher Full Text

Storm L, Tressoldi PE, Utts J: Testing the Storm et al. (2010) meta-analysis using Bayesian and frequentist approaches: Reply to Rouder et al. (2013) Psychol Bull. 2013; 139(1): 248-254.

PubMed Abstract | Publisher Full Text

Storm L, Tressoldi P: Meta-Analysis of Free-Response Studies 2009-2018:
Assessing the Noise-Reduction Model Ten Years On. PsyArxiv. 2020.

Publisher Full Text

Tressoldi P, Storm L: Anomalous perception in a Ganzfeld condition: A metaanalysis of more than 40 years investigation. figshare. Online resource. 2020. http://www.doi.org/10.6084/m9.figshare.12674618.v1

van Aert RCM, van Assen MALM: Correcting for publication bias in a MetaAnalysis with the P-Uniform* method. 2019.

Publisher Full Text

Viechtbauer W: The metafor Package. 2017.

Reference Source

Watt CA, Kennedy JE: Options for Prospective Meta-Analysis and Introduction of Registration-Based Prospective Meta-Analysis. Front Psychol. 2016; 7: 2030. PubMed Abstract | Publisher Full Text | Free Full Text 
The benefits of publishing with F1000Research:

- Your article is published within days, with no editorial bias

- You can publish traditional articles, null/negative results, case reports, data notes and more

- The peer review process is transparent and collaborative

- Your article is indexed in PubMed after passing peer review

- Dedicated customer support at every stage

For pre-submission enquiries, contact research@f1000.com 